\title{
Semiclassical quantization of non-Hamiltonian dynamical systems without memory
}

\author{
E. D. Vol \\ B. Verkin Institute for Low Temperature Physics and Engineering National \\ Academy of Sciences of Ukraine, Lenin av. 47 Kharkov 61103, Ukraine
}

\begin{abstract}
We propose a new method of quantization of a wide class of dynamical systems that originates directly from the equations of motion. The method is based on the correspondence between the classical and the quantum Poisson brackets, postulated by Dirac. This correspondence applied to open (non-Hamiltonian) systems allows one to point out the way of transition from the quantum description based on the Lindblad equation to the dynamical description of their classical analogs by the equations of motion and vice versa. As the examples of using of the method we describe the procedure of the quantization of three widely considered dynamical systems: 1) the harmonic oscillator with friction, 2) the oscillator with a nonlinear damping that simulates the process of the emergence of the limit cycle, and 3) the system of two periodic rotators with a weak interaction that synchronizes their oscillations. We discuss a possible application of the method for a description of quantum fluctuations in Josephson junctions with a strong damping and for the quantization of open magnetic systems with a dissipation and a pumping.
\end{abstract}

PACS numbers: 03.65.Ta

The main goal of this paper is to point out the close connection between the description of an open quantum system (OQS) in the Markovian approximation by the Lindblad equation and the description of its classical ana$\log (\mathrm{CA})$, if it exists, by equations of motion presented in a special form. To settle this connection in the easiest way one can use the correspondence principle (CP) between the classical and the quantum Poisson brackets. The principle was postulated by Dirac in his famous book [1]. Having this connection in hands we propose a new method of quantization of non-Hamiltonian systems without memory. The procedure of using of the method consists in the following. Let us assume that the quantum system of interest has the CA with known equations of motion. We also imply that these equations can be presented in a special form prescribed by the method - the form allowing the quantization (FAQ). In this case, on the base of the $\mathrm{CP}$ one can formulate an efficient recipe for finding the concrete form of the operators that give the quantum description of this system in the framework of the Lindblad equation. Below, we call such a procedure the semiclassical quantization of the dynamical systems (without memory). We should emphasize that, to a certain extent, the method of quantization proposed is a heuristic one. Therefore, with reference to concrete examples, we demonstrate in detail the specifics of its application. As such examples we consider three popular dynamical systems: 1) the harmonic oscillator with a friction; 2) the oscillator with a nonlinear damping that simulates the mechanism of the emergence of the limit cycle near the bifurcation point; and 3) the system of two periodic rotators with a weak interaction that synchronizes their oscillations. In our opinion, the latter example is in a direct relation with the description of the quantum noise in a Josephson junction with a strong damping. Besides, we give a simple generalization of the method proposed that allows one to apply it for the semiclassical quantization of open magnetic systems described by the equation of the Landau-Lifshitz type with a dissipation and under a pumping. Now, let us go to the details.

It is well-known that quantum mechanics in its original form (the Schrodinger equation for the wave function $\Psi$ of the system: $i \hbar \partial \Psi / \partial t=\hat{H} \Psi)$ provides the consistent description of the evolution of the state of a closed microscopic system. Such a description is extended automatically to the systems with a given dependence of their energy on time: $\hat{H}=\hat{H}(t)$. If a closed quantum system has the classical analog, the substitution of the semiclassical wave function $\Psi=A \exp \left(i S_{c l} / \hbar\right)$ into the Schrödinger equation allows one to obtain the well-known HamiltonJacobi equation for the action function $S_{c l}(q, t)$ (in the limit $\hbar \rightarrow 0$ ), see, e.g. [2]. In that way one turns from the quantum to the classical description of a closed system. If the microscopic system $S$ we are interested in is an open one, i.e. it interacts with an environment (a reservoir $R$ ) that, as a rule, contains a large number of degrees of freedom, the quantum mechanics yields the following recipe for the description of the evolution of the state of $S$. One should consider the composite system $S R$ that includes $S$ and the reservoir $R$ (that contains all exterior degrees of freedom with which the system $S$ interacts). The evolution of the density matrix of the closed system $\hat{\rho}_{S R}(t)$ is described by the quantum Liouville - von Neumann equation:

$$
\frac{d \hat{\rho}_{S R}}{d t}=-\frac{i}{\hbar}\left[\hat{H}_{S R}, \hat{\rho}_{S R}\right]
$$

where $\hat{H}_{S R}=\hat{H}_{S}+\hat{H}_{R}+\hat{H}_{\text {int }}$ is the Hamiltonian of the closed system $S R$. In case when the solution of Eq. (1) is known, the desired density matrix $\hat{\rho}_{S}(t)$ is found by the reduction of $\hat{\rho}_{S R}(t)$ over the variables that describe the reservoir: $\hat{\rho}_{S}(t)=\operatorname{tr}_{R} \hat{\rho}_{S R}(t)$. As a rule, for real systems with interaction this general recipe is unrealizable. Therefore, most often, the procedure of reduction is applied directly to the the equation (II) and then one tries to obtain a closed equation for $\hat{\rho}_{S}(t)$ using 
approximations based on the presence of a small parameter in a concrete physical system. On this way a large number of interesting and important results for quantum systems with dissipation were obtained (see [3] and references therein). Nevertheless, such an approach is not a universal one, and in many cases it cannot be realized. Regarding the standard approach one should note that it looks rather "uneconomical" from the point of view of the experiment, since the description of an open system $S$ requires the full information about the system $S R$. In connection with that, an alternative approach based on the quantum theory of open systems [4] becomes more and more popular during last years. In that approach, one attempts to describe the behavior of the OQS using only the information about the system $S$. The approach is based on the general restrictions imposed by the quantum mechanics on the evolution of $\hat{\rho}_{S}(t)$ : the linearity, the positivity, and the conservation of the trace of $\hat{\rho}_{S}(t)$. One of the most important results obtained in the framework of this approach is the Lindblad equation. This equation realizes the quantum scheme of the description of the evolution of the OQS in the Markovian approximation. The Lindblad equation for the evolution of the density matrix of OQS $\hat{\rho}_{S}(t)$ has the following form [5]:

$$
\frac{d \hat{\rho}_{S}}{d t}=-\frac{i}{\hbar}\left[\hat{H}, \hat{\rho}_{S}\right]+\sum_{j=1}^{N}\left\{\left[\hat{R}_{j} \hat{\rho}_{S}, \hat{R}_{j}^{+}\right]+\left[\hat{R}_{j}, \hat{\rho}_{S} \hat{R}_{j}^{+}\right]\right\}
$$

The first term in the right hand side of Eq. (21) describes the internal (Hamilton) dynamics of $S$, while the other terms model its interaction with the reservoir $R$ ( $N$ is the number of channels of the interaction of $S$ with $R$ ). We emphasize that the information determined in the standard approach by the structure of the reservoir and by the type of the interaction between $S$ and $R$ is included now in the operators $\hat{R}_{j}, \hat{R}_{j}^{+}$that acts in the space of states of the system $S$.

As an example, let us consider the situation when $N=$ 1 and the operator $\hat{R}$ is the Hermitian one $\hat{R}=\hat{R}^{+}=\hat{O}$. It turns out that in this important special case Eq. (2) describes the decoherence of the quantum system interacting with the "meter" (macroscopic or mesoscopic one) that measures continuously the observable $\hat{O}$, see $[\underline{6}$.

Let us assume now that there exists a quantum system $S$ (of the Markov type) which is described by Eq. (2) with some $\hat{H}, \hat{R}_{j}$ and $\hat{R}_{j}^{+}$, and the system has the classical analog. The question emerges: how can one find the dynamics, i.e. the equations of motion, of that $\mathrm{CA}$ from the Lindblad equation for $S$ ? The answer on this question can be obtained with the help of the correspondence principle formulated by Dirac in [1]. According to Dirac, the quantum Poisson bracket (commutator) of two operators $\hat{A}$ and $\hat{B}$ is connected with the Poisson bracket $\{A, B\}$ of their CA's - $A\left(q_{r}, p_{r}\right)$ and $B\left(q_{r}, p_{r}\right)$ by the relation

$$
[\hat{A}, \hat{B}]=i \hbar\{A, B\}
$$

where, by definition,

$$
\{A, B\}=\sum_{r} \frac{\partial A}{\partial q_{r}} \frac{\partial B}{\partial p_{r}}-\frac{\partial A}{\partial p_{r}} \frac{\partial B}{\partial q_{r}} .
$$

For the simplest operators $\hat{A}$ and $\hat{B}$ (for instance, $\hat{A}=\hat{q}_{r}$ and $\hat{B}=\hat{p}_{r}$ ) the relation (3) is exact one. In other cases it is fulfilled approximately, at least, in the first order in $\hbar$. Let us use this CP for finding the classical analog of the Lindblad equation. We imply that in the limit $\hbar \rightarrow 0$ the distribution function $f_{S}\left(q_{r}, p_{r}\right)$ in the phase space of the system $S$ corresponds to the density matrix $\hat{\rho}_{S}$. We will consider the simplest case, when $S$ has one degree of freedom and $N$, the number of channels of the interaction of $S$ and $R$, is equal to one. Let the operators $\hat{H}, \hat{R}$ and $\hat{R}^{+}$in Eq. (2) be the functions of the operators $\hat{q}$ and $\hat{p} \equiv(\hbar / i) \partial / \partial q$. Then, according to Eqs. (2) and (3), one can write the following equation for the distribution function of $\mathrm{CA}$ of the system $\mathrm{S}$ :

$$
\frac{\partial f_{S}}{\partial t}=\left\{H, f_{S}\right\}+i \hbar\left\{R f_{S}, \bar{R}\right\}+i \hbar\left\{R, f_{S} \bar{R}\right\}
$$

where $H(p, q), \quad R(p, q)$ and $\bar{R}(p, q)$ are the classical analogs of $\hat{H}, \hat{R}$ and $\hat{R}^{+}$, correspondingly. Using the definition (4) and differentiating explicitly, we reduce Eq. (5) to the following form:

$$
\frac{\partial f_{S}}{\partial t}+A(q, p) \frac{\partial f_{S}}{\partial q}+B(q, p) \frac{\partial f_{S}}{\partial p}+C(q, p) f_{S}=0
$$

where the notations

$$
\begin{array}{r}
A(q, p)=\frac{\partial H}{\partial p}+i \hbar\left(\bar{R} \frac{\partial R}{\partial p}-R \frac{\partial \bar{R}}{\partial p}\right) \\
B(q, p)=-\frac{\partial H}{\partial q}+i \hbar\left(R \frac{\partial \bar{R}}{\partial q}-\bar{R} \frac{\partial R}{\partial q}\right) \\
C(q, p)=2 i \hbar\left(\frac{\partial R}{\partial p} \frac{\partial \bar{R}}{\partial q}-\frac{\partial R}{\partial q} \frac{\partial \bar{R}}{\partial p}\right)
\end{array}
$$

are used.

The first order partial differential equation (6) describes the motion of the ensemble of systems (the phase liquid) in the space $\Gamma_{S} \equiv(q, p)$ of the system $S$. It is known (see []]) that the phase points (elements of the ensemble) move along the characteristics of Eq. (6) according to the equations of motion:

$$
\begin{gathered}
\dot{q} \equiv A(q, p)=\frac{\partial H}{\partial p}+i \hbar\left(\bar{R} \frac{\partial R}{\partial p}-R \frac{\partial \bar{R}}{\partial p}\right) \\
\dot{p} \equiv B(q, p)=-\frac{\partial H}{\partial q}+i \hbar\left(R \frac{\partial \bar{R}}{\partial q}-\bar{R} \frac{\partial R}{\partial q}\right)
\end{gathered}
$$

Now, let us compute the divergence of the phase velocity $\mathbf{v}=(\dot{q}, \dot{p})$. By definition

$$
\operatorname{div} \mathbf{v}=\frac{\partial \dot{q}}{\partial q}+\frac{\partial \dot{p}}{\partial p}=2 i \hbar\left(\frac{\partial \bar{R}}{\partial q} \frac{\partial R}{\partial p}-\frac{\partial R}{\partial q} \frac{\partial \bar{R}}{\partial p}\right)
$$


Using the relations (8) and (9) we find that Eq. (6) is reduced to the standard form of the continuity equation of motion for the phase liquid in the space $\Gamma_{S}$

$$
\frac{\partial f_{S}}{\partial t}+\operatorname{div}\left(f_{S} \mathbf{v}\right)=0
$$

Thus, with the help of the correspondence principle (3) we arrive to the conclusion that in the limit $\hbar \rightarrow 0$ the Lindblad equation (2) turns to the continuity equation for the distribution function $f_{S}$ for the CA of the system $S$. The equations of motion for that CA (Eq. (8)) follow directly from Eq. (2). This is the main result of the paper and we will use it for solving the inverse problem: the obtaining of quantum description of the dynamical system (without memory) from the equations of motion for its CA.

It is convenient to present the equations of motion (8) in the form of one complex equation for the complex coordinate. Let us define the complex variables $z$ and $z^{*}$ :

$$
z=\frac{\frac{q}{l_{0}}+\frac{i p l_{0}}{\hbar}}{\sqrt{2}}, \quad z^{*}=\frac{\frac{q}{l_{0}}-\frac{i p l_{0}}{\hbar}}{\sqrt{2}},
$$

where $l_{0}$ is an arbitrary parameter with the dimension of the length. It follows from the definition (11) that for any choice of $l_{0}$ the Poisson bracket of the dimensionless variables $z$ and $z^{*}$ is

$$
\left\{z, z^{*}\right\}=-\frac{i}{\hbar}\{q, p\}=-\frac{i}{\hbar} .
$$

Using the system of equations of motion (8) for $q$ and $p$, we find the desired dynamical equation for $z$ :

$$
\frac{d z}{d t}=-i \frac{\partial H}{\partial z^{*}}+\left(\bar{R} \frac{\partial R}{\partial z^{*}}-R \frac{\partial \bar{R}}{\partial z^{*}}\right) .
$$

It is implied here that the functions $H(q, p), R(q, p)$ and $\bar{R}(q, p)$ in Eq. (13) are expressed as the functions on $z$ and $z^{*}$ with the help of relations inverse to Eq. (11):

$$
q=\frac{l_{0}}{\sqrt{2}}\left(z^{*}+z\right), \quad p=\frac{i \hbar}{\sqrt{2} l_{0}}\left(z^{*}-z\right) .
$$

The equation of motion for the complex coordinate $z$ written in the form (13) is referred as the one presented in the form allowed the quantization (FAQ). Now, let us give the recipe (based on the $\mathrm{CP}$ ) for the transition from the description of the CA of the system $S$ by the equation of motion to the quantum description of $S$ by the Lindblad equation. The recipe proposed consists of three consecutive steps.

1. The input dynamical equations should be presented in the FAQ written for the complex coordinate $z$ in the form (13). Such a representation determines automatically the functions $H\left(z, z^{*}\right), R\left(z, z^{*}\right)$ and $\bar{R}\left(z, z^{*}\right)$ entered in the FAQ.

2. One finds the quantum analogs $\hat{H}, \hat{R}$ and $\hat{R}^{+}$of that functions. For that one should replace the coordinates $z$ and $z^{*}$ to the corresponding operators $\hat{z}$ and $\hat{z}^{+}$.
According to the CP (see (3)), $\left[\hat{z}, \hat{z}^{+}\right]=i \hbar\left\{z, z^{*}\right\}=1$. The latter relation allows one to identify the operators $\hat{z}$ and $\hat{z}^{+}$with the standard Bose operators $\hat{a}$ and $\hat{a}^{+}$, that satisfy $\left[\hat{a}, \hat{a}^{+}\right]=1$. We note that in the case when the polynomial functions $H, R$ and $\bar{R}$ of $z$ and $z^{*}$ contain the terms of the form $z^{k}\left(z^{*}\right)^{l}$ the ordering of the operators $\hat{a}$ and $\hat{a}^{+}$should be fixed and necessary symmetrization should be fulfilled under the transition to the quantum analogs $\hat{H}, \hat{R}$ and $\hat{R}^{+}$. Below we demonstrate how this problem is resolved with reference to concrete examples. Note, that the same problem emerges under the quantization of the Hamilton systems, as well.

3. The operators $\hat{H}, \hat{R}$ and $\hat{R}^{+}$should be substituted into the Lindblad equation (2) for the evolution of the density matrix. The correspondence principle guarantees that the equation obtained by this method will give a correct quantum description of the evolution of $S$, at least, with the accuracy up to the first order in $\hbar$. Therefore, the procedure described can be referred as the method of the semiclassical quantization of dynamical systems (without memory). We note that the most delicate point of the method is the presentation of the equations of motion in the FAQ - it is difficult to formalize this point.

The rest of the paper is devoted to the study of concrete physical systems that can be quantized by the method proposed.

\section{Example A. Harmonic oscillator with friction}

This simplest example of a quantum dissipative system was considered more than once in the literature. In particular, in the recent review by Mensky [8] the equation of the Lindblad type for the density matrix of such an oscillator was obtained by the method of restricted Feynman integrals (the method of quantum corridors). Let us show how simple the derivation of this equation (up to the notations) by the method proposed here. The equation of motion for the harmonic oscillator with friction has the well-known form:

$$
m \ddot{q}+2 \gamma \dot{q}+m \omega_{0}^{2} q=0,
$$

where $\gamma$ is the friction coefficient, $\omega_{0}$, the oscillatory frequency, and m, its mass. Eq. (15) can be presented as the system of two first order equations:

$$
\begin{array}{r}
\dot{q}=\frac{p}{m}, \\
\dot{p}=-\frac{2 \gamma}{m} p-m \omega_{0}^{2} q .
\end{array}
$$

Let us consider the complex coordinate $z=\left(q / l_{0}+\right.$ $\left.i p l_{0} / \hbar\right) / \sqrt{2}$. In this problem it is convenient to choose the oscillatory length as the parameter $l_{0}=\sqrt{\hbar / m \omega_{0}}$. At such $l_{0}$, using Eq. (16), we obtain the equation of motion for $z$ in the form:

$$
\frac{d z}{d t}=-i \omega_{0} z-\lambda\left(z-z^{*}\right)
$$


where the notation $\lambda \equiv \gamma / m$ is introduced.

Let us show that Eq. (17) can be presented in the FAQ. We define two functions of the variables $z$ and $z^{*}$ : the real one $H=\hbar \omega_{0}|z|^{2}+i \hbar \lambda\left[(z *)^{2}-z^{2}\right] / 2$ and the complex one $R=\sqrt{\lambda}\left(z \cosh u-z^{*} \sinh u\right)$, where $u$ is an arbitrary parameter. Then we check directly that the right hand side of Eq. (18) can be written in the form

$$
-i \omega_{0} z-\lambda\left(z-z^{*}\right)=-\frac{i}{\hbar} \frac{\partial H}{\partial z^{*}}+\left(\bar{R} \frac{\partial R}{\partial z^{*}}-R \frac{\partial \bar{R}}{\partial z^{*}}\right) .
$$

Therefore, in accordance with the method proposed, the quantum description of the harmonic oscillator with friction is given by the Lindblad equation

$$
\frac{d \hat{\rho}}{d t}=-\frac{i}{\hbar}[\hat{H}, \hat{\rho}]+\left[\hat{R} \hat{\rho}, \hat{R}^{+}\right]+\left[\hat{R}, \hat{\rho} \hat{R}^{+}\right]
$$

where the quantum analogs of the functions $H\left(z, z^{*}\right)$ and $R\left(z, z^{*}\right)$ are determined as

$$
\begin{gathered}
\hat{H}=\hbar \omega_{0} \hat{a}^{+} \hat{a}+\frac{i \hbar \lambda}{2}\left[\left(\hat{a}^{+}\right)^{2}-\hat{a}^{2}\right], \\
\hat{R}=\sqrt{\lambda}\left(\hat{a} \cosh u-\hat{a}^{+} \sinh u\right) .
\end{gathered}
$$

Equation (19) together with the expressions for $\hat{H}$ and $\hat{R}$ (Eq. (20) coincides with the result Eq. (51) of Ref. [8] up to the notations. Using Eqs. (19) and (20), one can derive the equations of motion for the first $(\overline{\hat{q}}, \overline{\hat{p}})$ and the second $\left(\overline{\hat{q}^{2}}, \overline{\hat{p}^{2}}, \overline{\hat{q} \hat{p}+\hat{p} \hat{q}}\right)$ momenta of $\hat{q}$ and $\hat{p}$ - the basic physical quantities of the system $S$. But we will not do that here, since Ref. [8] contains such a derivation and the subsequent analysis (including a discussion on the physical meaning of the parameter $u$ ). We have considered the harmonic oscillator with only one aim: to illustrate all the specifics of the method of quantization on the example of the simplest model. Let us now turn to more interesting examples. To simplify the notations, we will use the system of units with $\hbar=1$. Also, the dimensionless coordinates $x \equiv q / l_{0}$ and $y \equiv p l_{0} / \hbar$ will be used instead of the variables $q$ and $p$. Consequently, the complex coordinate $z$ in the FAQ is defined as $z=(x+i y) / \sqrt{2}$.

\section{Example B. Quantum analog of the bifurcation of the emergence of the cycle}

Let us show how the quantum description of the oscillator with a nonlinear damping can be given by the method proposed. The $\mathrm{CA}$ of such an oscillator simulates the mechanism of a smooth emergence of auto-oscillations near the bifurcation point. Similar bifurcation of the emergence of the cycle is typical for the situation when an equilibrium point losses its stability under a variation of the parameters of the dynamical system. In such a situation the growing up of small oscillations may switch the system to a new stationary state that corresponds to the closed trajectory (limit cycle). The following system of equations gives the mathematical model of the behavior of the dynamical system near the bifurcation of the emergence of the cycle (see [9]):

$$
\begin{array}{r}
\frac{d x}{d t}=\omega y+\lambda x-\mu x\left(x^{2}+y^{2}\right), \\
\frac{d y}{d t}=-\omega x+\lambda y-\mu y\left(x^{2}+y^{2}\right) .
\end{array}
$$

The system of the equations of motion Eq. (21) can be written in the complex form:

$$
\frac{d z}{d t}=-i \omega z+\lambda z-2 \mu z|z|^{2}
$$

Equation (22) can be presented in the FAQ. To show this we introduce the functions $H=\hbar \omega z^{*} z, R_{1}=\sqrt{\lambda} z^{*}$ and $R_{2}=\sqrt{\mu} z^{2}$. Then we check directly that the right hand side of Eq. (22) can be rewritten in the form

$$
\begin{array}{r}
-i \omega z+\lambda z-2 \mu z|z|^{2}=-i \frac{\partial H}{\partial z^{*}} \\
+\left(\bar{R}_{1} \frac{\partial R_{1}}{\partial z^{*}}-R_{1} \frac{\partial \bar{R}_{1}}{\partial z^{*}}\right)+\left(\bar{R}_{2} \frac{\partial R_{2}}{\partial z^{*}}-R_{2} \frac{\partial \bar{R}_{2}}{\partial z^{*}}\right) .
\end{array}
$$

According to the recipe of quantization described above, the Lindblad equation for the evolution of the density matrix of the quantum analog of the system (21) can be written in the form

$$
\begin{aligned}
\frac{d \hat{\rho}}{d t}=-i[\hat{H}, \hat{\rho}] & +\left[\hat{R}_{1} \hat{\rho}, \hat{R}_{1}^{+}\right]+\left[\hat{R}_{1}, \hat{\rho} \hat{R}_{1}^{+}\right] \\
& +\left[\hat{R}_{2} \hat{\rho}, \hat{R}_{2}^{+}\right]+\left[\hat{R}_{2}, \hat{\rho} \hat{R}_{2}^{+}\right]
\end{aligned}
$$

with $\hat{H}=\omega \hat{a}^{+} \hat{a}, \hat{R}_{1}=\sqrt{\lambda} \hat{a}^{+}$, and $\hat{R}_{2}=\sqrt{\mu} \hat{a}^{2}$.

From physical reasons it is clear that the motion of the system (21) along the limit cycle should correspond to the stationary solution of Eq. (24) $\hat{\rho}_{s t}$, such that $d \hat{\rho}_{s t} / d t=0$. By analogy with the classical solution, for which $|z|^{2} \equiv$ const, one can expect that $\hat{\rho}_{s t}$ will commute with the particle number operator $\hat{N}=\hat{a}^{+} \hat{a}$. Therefore, we will seek the stationary solution of Eq. (24) in the form $\hat{\rho}_{s t}=$ $\sum_{n=0}^{\infty}|n\rangle \rho_{n}\langle n|$, where $|n\rangle$ are the eigenfunctions of the operator $\hat{N}$. Positive coefficients $\rho_{n}$ satisfy the additional normalization condition $\sum_{n=0}^{\infty} \rho_{n}=1$. Substituting this expansion into Eq. (24) we obtain the system of recurrent relations for the coefficients $\rho_{n}$ :

$$
\begin{array}{r}
2 \lambda\left[n \rho_{n-1}-(n+1) \rho_{n}\right]+ \\
2 \mu\left[(n+2)(n+1) \rho_{n+2}-n(n-1) \rho_{n}\right]=0 .
\end{array}
$$

It is convenient to introduce the generating function for the coefficients $\rho_{n}$. By definition $G(u) \equiv \sum_{n=0}^{\infty} \rho_{n} u^{n}$. One can check by the direct substitution that the system (25) is equivalent to the following second order differential equation for the function $G(u)$ :

$$
(1+u) \frac{d^{2} G}{d u^{2}}-\nu u \frac{d G}{d u}-\nu G(u)=0
$$

In Eq. (26) the notation $\nu=\lambda / \mu$ is used. 
Let us transform Eq. (26) to the canonical form. To do this we introduce the auxiliary variable $v=\nu(1+u)$. Then $d / d u=\nu d / d v$, and Eq. (26) becomes

$$
v \frac{d^{2} G}{d v^{2}}+(\nu-v) \frac{d G}{d v}-G(v)=0 .
$$

Eq. (27) coincides with the equation for the confluent hypergeometric function $y=\Phi(a, c, x)$ :

$$
x \frac{d^{2} y}{d x^{2}}+(c-x) \frac{d y}{d x}-a y(x)=0
$$

(see [10]) if one sets $c=\nu$ and $a=1$.

Therefore, the solution of Eq. (26) that satisfies all conditions of the problem can be written in the form

$$
G(u)=\frac{\Phi(1, \nu, \nu(1+u))}{\Phi(1, \nu, 2 \nu)} .
$$

In the solution Eq. (29) we take into account that the normalization condition $\sum_{n=0}^{\infty} \rho_{n}=1$ is equivalent to the relation $G(1)=1$ for the generating function $G(u)$.

Differentiating the expression Eq. (29) one can find the average values of all physical quantities of interest in the stationary state. For instance, the average number of quanta $\bar{n}$ generated in the stationary state is given by the relation

$$
\bar{n}=\sum_{n=0}^{\infty} n \rho_{n}=\left.\frac{d G(u)}{d u}\right|_{u=1},
$$

and the quantity $\overline{n^{2}}-\bar{n}=\overline{(n-1) n}$ is connected with the second derivative of $G(u)$ by the relation

$$
\overline{n^{2}}-\bar{n}=\left.\frac{d^{2} G(u)}{d u^{2}}\right|_{u=1} .
$$

To characterize the properties of the distribution of the number of quanta $\left\{\rho_{n}\right\}$ we will use the Mandel parameter $Q$ defined as (see [1])

$$
Q=\frac{\overline{n^{2}}-\bar{n}^{2}}{\bar{n}}-1=\frac{\overline{n(n-1)}}{\bar{n}}-\bar{n} .
$$

The distributions for which $Q>0$ are called the superPoisson ones, while the distribution with $Q<0$, the sub-Poisson ones. The Poisson distribution corresponds to $Q=0$. Using the properties of the confluent hypergeometric function, one can easily find that at $\nu=1$ the expression Eq. (29) corresponds to the Poisson distribution with $\bar{n}=1$, at $\nu>1$ it describes the super-Poisson distribution, and at $\nu<1$, the sub-Poisson distribution. We note also that for integer $\nu$ the generating function $G(u)$ can be presented through elementary functions. For instance, at $\nu=1$ it reads as $G(u)=e^{u-1}$, at $\nu=2$, $G(u)=(2 / \sinh 2)(\sinh (1+u) /(1+u)) e^{u-1}$ etc. Thus, the method proposed allows one to describe in detail the stationary state of OQS which classical analog simulates the bifurcation that occurs very frequently in the nonlinear mechanics.
Example C. Quantum analog of the phase synchronization of two auto-oscillating rotators

In this part of the paper we consider the specifics of application of the method for the problem of quantization of dynamical systems with several degrees of freedom. While such a generalization looks quite obvious, nevertheless, additional interesting opportunities emerge, that will be analyzed in detail. As a distinctive example that is of interest by itself, we consider the dynamical system of two interacting auto-oscillating rotators. In the case when their frequencies (in the absence of the interaction) are rather close to each other the switching on the interaction may result in the effect of synchronization, i.e. the locking of the frequencies and the phases in the bound system. The classical description of the effect of synchronization is well-known 12. Here we are interested in the possibility of its quantum description. We restrict the consideration by the case of weak interaction between the periodic oscillators, when the method of phase dynamics (see 12]) can be used for their description. For the simplest form of the coupling between the oscillators this method yields the following system of equations of motion for their phases $\varphi_{1}$ and $\varphi_{2}$

$$
\begin{aligned}
& \frac{d \varphi_{1}}{d t}=\omega_{1}+a \sin \left(\varphi_{2}-\varphi_{1}\right), \\
& \frac{d \varphi_{2}}{d t}=\omega_{2}+a \sin \left(\varphi_{1}-\varphi_{2}\right),
\end{aligned}
$$

where $\omega_{1}\left(\omega_{2}\right)$ is the frequency of motion the first (second) oscillator in its limit cycle, and $a$ is the coupling constant that determines the strength of the interaction between the oscillators.

It is implied in this approximation that each oscillator (rotator) moves along the limit cycle with a constant amplitude, i.e., the additional restrictions for the system Eq. (33) $\left(d r_{1} / d t=d r_{2} / d t=0\right)$ are assumed. Taking into account these restrictions explicitly, one can write the system Eq. (33) in the following equivalent form:

$$
\begin{array}{r}
\frac{d \varphi_{1}}{d t}=\omega_{1}+\lambda r_{1} r_{2} \sin \left(\varphi_{2}-\varphi_{1}\right), \\
\frac{d \varphi_{2}}{d t}=\omega_{2}+\lambda r_{1} r_{2} \sin \left(\varphi_{1}-\varphi_{2}\right), \\
\frac{d r_{1}}{d t}=\frac{d r_{2}}{d t}=0 .
\end{array}
$$

The system Eq. (34) can be presented in the form of two dynamical equations for the complex coordinates $z_{1}=$ $\left(x_{1}+i y_{1}\right) / \sqrt{2}=r_{1} e^{i \varphi_{1}} / \sqrt{2}$ and $z_{2}=\left(x_{2}+i y_{2}\right) / \sqrt{2}=$ $r_{2} e^{i \varphi_{2}} / \sqrt{2}$ :

$$
\begin{aligned}
& \frac{d z_{1}}{d t}=i \omega_{1} z_{1}+\lambda z_{1}\left(z_{1}^{*} z_{2}-z_{2}^{*} z_{1}\right), \\
& \frac{d z_{2}}{d t}=i \omega_{2} z_{2}+\lambda z_{2}\left(z_{2}^{*} z_{1}-z_{1}^{*} z_{2}\right) .
\end{aligned}
$$

Indeed, if one substitutes the expressions for $z_{1}$ and $z_{2}$ into Eq. (35) and then separates the real and the imaginary parts, one obtains just the system (35). Let us show 
that Eq. (35) can be presented in the FAQ. To do this we choose the following functions $H$ and $R$ :

$$
\begin{array}{r}
H=-\omega_{1}\left|z_{1}\right|^{2}-\omega_{2}\left|z_{2}\right|^{2}+\frac{i \lambda\left|z_{1}\right|^{2}}{2}\left(z_{1}^{*} z_{2}-z_{2}^{*} z_{1}\right) \\
+\frac{i \lambda\left|z_{2}\right|^{2}}{2}\left(z_{2}^{*} z_{1}-z_{1}^{*} z_{2}\right), \\
R=\frac{\sqrt{\lambda}}{2}\left(\left|z_{1}\right|^{2}-\left|z_{2}\right|^{2}+z_{2}^{*} z_{1}-z_{2} z_{1}^{*}\right) .
\end{array}
$$

One can check directly that the right hand sides of the equations of the system (35) are expressed as

$$
\begin{array}{r}
i \omega_{1} z_{1}+\lambda z_{1}\left(z_{1}^{*} z_{2}-z_{2}^{*} z_{1}\right)=-i \frac{\partial H}{\partial z_{1}^{*}} \\
+\left(\bar{R} \frac{\partial R}{\partial z_{1}^{*}}-R \frac{\partial \bar{R}}{\partial z_{1}^{*}}\right), \\
i \omega_{2} z_{2}+\lambda z_{2}\left(z_{2}^{*} z_{1}-z_{1}^{*} z_{2}\right)=-i \frac{\partial H}{\partial z_{2}^{*}} \\
+\left(\bar{R} \frac{\partial R}{\partial z_{2}^{*}}-R \frac{\partial \bar{R}}{\partial z_{2}^{*}}\right) .
\end{array}
$$

Then, following the method of quantization proposed, one can pass to the corresponding Lindblad equation in which the operators $\hat{H}$ and $\hat{R}$ are obtained from the functions (36) by the substitution $z_{1} \rightarrow \hat{a}_{1}, z_{1}^{*} \rightarrow \hat{a}_{1}^{+}$, $z_{2} \rightarrow \hat{a}_{2}, z_{2}^{*} \rightarrow \hat{a}_{2}^{+}$. But, there is another formulation of this problem, that is especially useful for the quantization of open magnetic systems. Let us describe in short this formulation.

Let the dynamical systems with two degrees of freedom be determined by its equations of motion presented in FAQ with some functions $H$ and $R$

$$
\begin{aligned}
& \frac{d z_{1}}{d t}=-i \frac{\partial H}{\partial z_{1}^{*}}+\left(\bar{R} \frac{\partial R}{\partial z_{1}^{*}}-R \frac{\partial \bar{R}}{\partial z_{1}^{*}}\right), \\
& \frac{d z_{2}}{d t}=-i \frac{\partial H}{\partial z_{2}^{*}}+\left(\bar{R} \frac{\partial R}{\partial z_{2}^{*}}-R \frac{\partial \bar{R}}{\partial z_{2}^{*}}\right) .
\end{aligned}
$$

Instead of the complex coordinates $z_{1}$ and $z_{2}$ we introduce three real valued variables $l_{x}=\left(z_{1}^{*} z_{2}+z_{2}^{*} z_{1}\right) / 2$, $l_{y}=i\left(z_{2}^{*} z_{1}-z_{1}^{*} z_{2}\right) / 2$, and $l_{z}=\left(\left|z_{1}\right|^{2}-\left|z_{2}\right|^{2}\right) / 2$. We imply that the Poisson brackets for the coordinates $z_{1}, z_{1}^{*}$, $z_{2}$, and $z_{2}^{*}$ satisfy the relations $\left\{z_{\alpha}, z_{\beta}\right\}=0,\left\{z_{\alpha}^{*}, z_{\beta}^{*}\right\}=0$, and $\left\{z_{\alpha}, z_{\beta}^{*}\right\}=-i \delta_{\alpha \beta}$, where $\alpha, \beta=1,2$. Then it follows from the definition of $l_{x}, l_{y}$ and $l_{z}$ that the Poisson brackets for the components of the vector $\mathbf{l}=\left(l_{x}, l_{y}, l_{z}\right)$ have the form $\left\{l_{i}, l_{k}\right\}=\epsilon_{i j k} l_{k}(i, j, k=1,2,3)$. It allows one to identify the vector $\mathbf{l}$ with the angular momentum of the system considered. If $H$ and $R$ in Eq. (38) can be presented as functions of $l_{x}, l_{y}$ and $l_{z}$, then, substituting the variables and doing a simple algebra, we find that the system Eq.(38) is equivalent to the following equation of motion for the vector $\mathbf{l}$ :

$$
\frac{d \mathbf{l}}{d t}=-\left(\mathbf{l} \times \frac{\delta H}{\delta \mathbf{l}}\right)+i R\left(\mathbf{l} \times \frac{\delta \bar{R}}{\delta \mathbf{l}}\right)+c . c .
$$

Eq. (39) can be considered as a generalization of the standard equation of motion for the magnetic momentum
(Landau-Lifshitz equation) for the case of the momentum interacting with the environment. One can see from Eq. (39) that such an equation is valid under the condition of conservation of the total magnetic momentum under the motion. Physically, it means that the interaction of the open system with the environment is invariant with respect to rotations of the system. The representation of the equations of motion for the magnetic momentum in the form (39) with given $H(\mathbf{l}), R(\mathbf{l})$ and $\bar{R}(\mathbf{l})$ allows one to fulfill the obvious transition to the quantum description on the base of the Lindblad equation. To do this one should find the operators $\hat{H}, \hat{R}$ and $\hat{R}^{+}$, the quantum analogs of the functions $H, R$ and $\bar{R}$, correspondingly. As before, the procedure of finding of that operators is determined by the correspondence principle: one should replace the variables $l_{x}, l_{y}$ and $l_{z}$ in the expressions for $H, R$ and $\bar{R}$ to the operators $\hat{l}_{x}, \hat{l}_{y}$ and $\hat{l}_{z}$ that satisfy the commutation relations $\left[\hat{l}_{i}, \hat{l}_{j}\right]=i \epsilon_{i j k} \hat{l}_{k}$. Then, if necessary, the expressions obtained should be ordered and symmetrized.

Let us now show how this variant of the quantization method works in the problem we are interested in - the problem on the synchronization of two interacting rotators. First of all, we note that the quantities $H, R$ and $\bar{R}$ in Eq. (36) are expressed as functions of $l_{x}, l_{y}$ and $l_{z}$ as follows

$$
\begin{array}{r}
H=-\left(\omega_{1}-\omega_{2}\right) l_{z}-2 \lambda l_{y} l_{z}, \\
R=\sqrt{\lambda}\left(l_{z}-i l_{y}\right) .
\end{array}
$$

We omit the term $-\left(\omega_{1}+\omega_{2}\right)\left(\left|z_{1}\right|^{2}+\left|z_{2}\right|^{2}\right) / 2$ in the expression for $H$ in Eq. (40), because this term is the integral of motion of the equations (35).

The quantum description of the system we are interested in is given by the following Lindblad equation:

$$
\frac{d \hat{\rho}}{d t}=-\frac{i}{\hbar}[\hat{H}, \hat{\rho}]+\left[\hat{R} \hat{\rho}, \hat{R}^{+}\right]+\left[\hat{R}, \hat{\rho} \hat{R}^{+}\right],
$$

in which $\hat{H}$ and $\hat{R}$ are the quantum analogs of the functions $H$ and $R$ (Eq. (40)):

$$
\begin{array}{r}
H=-\left(\omega_{1}-\omega_{2}\right) \hat{l}_{z}-\lambda\left(\hat{l}_{y} \hat{l}_{z}+\hat{l}_{z} \hat{l}_{y}\right), \\
\hat{R}=\sqrt{\lambda}\left(\hat{l}_{z}-i \hat{l}_{y}\right) .
\end{array}
$$

Using the Lindblad equation (41) one can find the equation of motion for the average value of an arbitrary observable $\hat{A}: \overline{\hat{A}} \equiv \operatorname{tr} \hat{\rho} \hat{A}$. The direct calculation of $\overline{d \hat{A}} / d t$ yields the following result:

$$
\frac{d \overline{\hat{A}}}{d t}=-i \overline{[\hat{A}, \hat{H}]}+\overline{\hat{R}^{+}[\hat{A}, \hat{R}]}+\overline{\left[\hat{R}^{+}, \hat{A}\right] \hat{R}}
$$

Using Eq. (43) we obtain the system of equations of motion for the first order momenta $\overline{\hat{l}_{x}}, \bar{l}_{y}$ and $\overline{\hat{l}_{z}}$ :

$$
\frac{d \overline{\hat{l}_{x}}}{d t}=-2 \lambda \overline{\hat{l}_{x}}+4 \lambda \overline{\hat{l}_{y}^{2}}+\delta \overline{\hat{l}_{y}}
$$




$$
\begin{aligned}
& \frac{d \hat{l}_{y}}{d t}=-\lambda \hat{l}_{y}-2 \lambda\left(\overline{\hat{l}_{x} \hat{l}_{y}+\hat{l}_{y} \hat{l}_{x}}\right)-\delta \overline{\hat{l}_{x}}, \\
& \frac{d \hat{l}_{z}}{d t}=-\lambda \overline{\hat{l}_{z}} .
\end{aligned}
$$

In Eq. (44) we introduce the notation $\delta \equiv \omega_{1}-\omega_{2}$ for the difference of the frequencies of two rotators.

One can see from Eq. (44) that the equations of motion for the first order momenta contain the second order momenta $\hat{l}_{y}^{2}$ and $\hat{l}_{x} \hat{l}_{y}+\hat{l}_{y} \hat{l}_{x}$. Writing the equations of motion for the second order momenta

$$
\begin{aligned}
& \frac{d \hat{l}_{y}^{2}}{d t}=2 \lambda\left(\overline{\hat{l}_{x}^{2}}-\overline{\hat{l}_{z}^{2}}\right)-\delta\left(\overline{\hat{l}_{y} \hat{l}_{x}+\hat{l}_{x} \hat{l}_{y}}\right), \\
& \frac{d \hat{l}_{z}^{2}}{d t}=-2 \lambda \overline{\hat{l}_{y}\left(\hat{l}_{x} \hat{l}_{y}+\hat{l}_{y} \hat{l}_{x}\right)} \\
& -2 \lambda \overline{\left(\hat{l}_{x} \hat{l}_{y}+\hat{l}_{y} \hat{l}_{x}\right) \hat{l}_{y}}+2 \lambda\left(\overline{\hat{l}_{x}^{2}}-\overline{\hat{l}_{y}^{2}}\right), \\
& \frac{d\left(\overline{\hat{l}_{x} \hat{l}_{y}+\hat{l}_{y} \hat{l}_{x}}\right)}{d t}=8 \lambda \overline{\hat{l}_{y}^{3}}-3 \lambda\left(\overline{\hat{l}_{y} \hat{l}_{x}^{2}+\hat{l}_{x}^{2} \hat{l}_{y}}\right) \\
& -5 \lambda\left(\frac{\overline{\hat{l}_{y}} \hat{l}_{x}+\hat{l}_{x} \hat{l}_{y}}{)}-2 \lambda \overline{\hat{l}_{x} \hat{l}_{y} \hat{l}_{z}}+2 \delta\left(\overline{\hat{l}_{y}^{2}}-\bar{l}_{x}^{2}\right)-\lambda \bar{l}_{y}(45\right.
\end{aligned}
$$

we note that they contain the third order momenta, etc. Thus, further simplifications are needed for obtaining the solution of the quantum problem. Below, we consider only stationary states of the system (for which the left hand sides of Eqs. (44) and (45) are zero) and specify the case of complete synchronization $\delta=0$. To obtain the closed system of equation for the first order momenta and the second order momenta we decouple the third order momenta applying the approximation frequently used in such problems (see [13]):

$$
\overline{\hat{A} \hat{B} \hat{C}} \approx \overline{\hat{A} \hat{B}} \cdot \overline{\hat{C}}+\overline{\hat{A}} \cdot \overline{\hat{B} \hat{C}}+\overline{\hat{A} \hat{C}} \cdot \overline{\hat{B}}-2 \overline{\hat{A}} \cdot \overline{\hat{B}} \cdot \overline{\hat{C}} .
$$

As a final result, we obtain the following system of equations for the first and the second order momenta

$$
\begin{aligned}
& \overline{\hat{l}_{z}}=0, \quad \overline{\hat{l}_{x} \hat{l}_{y}}=\overline{\hat{l}_{y} \hat{l}_{x}}=-\frac{\overline{l_{y}}}{4} \\
& \overline{\hat{l}_{y}^{2}}=\frac{\overline{\hat{l}_{x}}}{2}, \quad \overline{\hat{l}_{x}^{2}}=\overline{\hat{l}_{z}^{2}} \\
& 4\left[2 \overline{\hat{l}_{y}} \cdot \overline{\hat{l}_{x} \hat{l}_{y}}+\overline{\hat{l}_{x}} \cdot \overline{\hat{l}_{y}^{2}}-2 \overline{\hat{l}_{x}} \cdot\left(\overline{\hat{l}_{y}}\right)^{2}\right]+\left(\overline{\hat{l}_{y}^{2}}-\overline{\hat{l}_{x}^{2}}\right)=0 \\
& 8\left[3 \overline{\hat{l}_{y}^{2}} \cdot \overline{\hat{l}_{y}}-2\left(\overline{\hat{l}_{y}}\right)^{3}\right]-8\left[2 \overline{\hat{l}_{x} \hat{l}_{y}} \cdot \overline{\hat{l}_{x}}+\overline{\hat{l}_{y}} \cdot \overline{\hat{l}_{x}^{2}}\right. \\
& \left.-2 \overline{\hat{l}_{y}}\left(\overline{\hat{l}_{x}}\right)^{2}\right]-10 \overline{\hat{l}_{x} \hat{l}_{y}}-\overline{\hat{l}_{y}}=0 .
\end{aligned}
$$

It follows from the last equation of the system (46) that $\overline{\hat{l}_{y}}=0$. This relation is valid also for the CA. Let us remind that in the classical limit $(\hbar \rightarrow 0) \hat{l}_{y} \rightarrow l_{y}=$ $r_{1} r_{2} \sin \left(\varphi_{2}-\varphi_{1}\right) \rightarrow 0$, since for the case of complete synchronization the phase difference $\varphi=\varphi_{2}-\varphi_{1} \rightarrow 0$. But, in difference with the CA, where $\overline{l_{y}^{2}}=r_{1}^{2} r_{2}^{2} \overline{\sin ^{2} \varphi}$ is also approaches zero, in the quantum case $\overline{l_{y}^{2}} \neq 0$ for the stationary state, and this quantity can be the measure of the quantum noise of the system. Let us find the value of $\overline{\hat{l}_{y}^{2}}$ using the system (46) and the angular momentum conservation law $\hat{l}_{x}^{2}+\hat{l}_{y}^{2}+\hat{l}_{z}^{2} \equiv \hat{L}^{2}=l(l+1)=(N / 2)(N / 2+1)$, where $\hat{N}=\hat{a}_{1}^{+} \hat{a}_{1}+\hat{a}_{2}^{+} \hat{a}_{2}$ is the total number of the excitations under the bosonic description of the system. Let us notate $x \equiv \overline{\hat{l}_{y}^{2}}$. It follows from (46) that $x=\overline{\hat{l}_{x}} / 2$ and it satisfies the simple equation

$$
8 x^{2}+\frac{3 x}{2}-\frac{N^{2}}{8}-\frac{N}{4}=0 \text {. }
$$

The solution of Eq. (47) is

$$
\overline{\hat{l}_{y}^{2}}=x=\frac{1}{8}\left[\left(N^{2}+2 N+\frac{9}{16}\right)^{1 / 2}-\frac{3}{4}\right] \approx \frac{N}{8} .
$$

In Eq. (48) we take into account that the semiclassical approximation requires the fulfilment of the inequality $N \gg 1$.

Let us summarize the main conclusions that concern the latter example.

1. The quantum description of the non-Hamiltonian system consisting of two rotators which oscillations be synchronized by their mutual interaction is possible not only with the use of standard Bose operators, but also in the representation, where the components of the operator of the angular momentum $\hat{\mathbf{l}}=\left(\hat{l}_{x}, \hat{l}_{y}, \hat{l}_{z}\right)$ be used as the generators.

2. Under the condition of complete synchronization $\left(\omega_{1}=\omega_{2}\right)$ the level of the quantum noise in this system is determined by the average value of the operator $\hat{l}_{y}^{2}$.

3. In the stationary state $\overline{\hat{l}_{y}^{2}} \simeq N / 8$, where $N$ is the total number of the excitations under the bosonic description of the system

It is necessary to note that the modification of the quantization method used in the latter example is based on the Schwinger representation 14] for the angular momentum operator in terms of two Bose operators.

To conclude this consideration, we discuss briefly the possibility of the application of the results of the last section to the study of quantum fluctuations in Josephson junctions with a strong damping. Usually, the dynamics of such a junction is described by the resistive model on the base of the following well-known equation [15]

$$
\frac{\hbar \dot{\varphi}}{2 e R}=I_{e x}-I_{c} \sin \varphi .
$$

In Eq. (49) the following notations are used: $\varphi=$ $\varphi_{1}-\varphi_{2}$ is the phase difference between the superconducting banks of the junction, $I_{e x}$, the input current applied to the banks, $R$, the Ohmic resistance of the junction, $I_{c}$, its critical current. Comparing Eqs. (49) and (33) we see that these equations coincide with each other up to the notations. The regime of the complete synchronization $\omega_{1}=\omega_{2}$ in the model of coupled rotators corresponds to the case of zero input current $I_{e x}$ in the Josephson junction model. As is easily seen, the parameter of the quantum noise $\overline{\hat{l}_{y}^{2}}$ is proportional to the average square of the 
bias voltage in the junction and this quantity can be measured experimentally. It is also obvious that the number of bosonic excitations $\hat{N}=\hat{a}_{1}^{+} \hat{a}_{1}+\hat{a}_{2}^{+} \hat{a}_{2}$ in the Josephson junction is equal to the number of Cooper pairs in its banks. In the framework of the Ginzburg-Landau theory this quantity is simply connected with the order param- eter of the superconductor [15]. The explicit form of the relation (48) for the Josephson junction and a detailed analysis of the quantum fluctuations by the method proposed will be given in further publications.

I would acknowledge L.A.Pastur for the discussion of the results of the paper and valuable comments.
[1] P.A.M. Dirac, The Principles of Quantum Mechanics, 4th ed., Clarendon Press, Oxford, 1958,

[2] L.D.Landau and E.M.Lifshitz, Quantum Mechanics, Pergamon Press, London, 1958.

[3] U. Weiss, Quantum Dissipative Systems, World Scientific, Singapore, 1999.

[4] E.B. Davies, Quantum Theory of Open Systems, Academic Press, London- New York, 1976.

[5] G.Lindblad, Commun. Math. Physics 48, 119 (1976).

[6] M.B. Mensky, Phys-Usp. 41, 923 (1998)[ Usp. Fiz. Nauk 168, 1019 (1998)].

[7] R. Courant, Partial Differential Equations, Interscience, New-York - London, 1962.

[8] M.B. Mensky, Phys-Usp. 46, 1163 (2003)[ Usp. Fiz. Nauk 173, 1199 (2003)].

[9] V.I.Arnold, Geometrical Methods in the Theory of Or- dinary Differential Equations, 2-nd ed., Springer, NewYork, 1988.

[10] H. Bateman and A. Erdelyi (eds.), Higher Transcendental Functions, v. 1, chap. 6, McGraw-Hill, New-York, 1953.

[11] M.O. Scully and M.S. Zubairy, Quantum Optics, Cambridge University Press, 1997.

[12] A. Pikovsky, M. Rosenblum, J. Kurths, Synchronization. An Universal Concept in Nonlinear Science, Cambridge University Press, 2002.

[13] A. Vardi, J. R. Anglin, Phys. Rev. Lett. 86, 568 (2001)

[14] J. Schwinger, in: Quantum theory of angular momentum, L.C. Biederharn and H. van Dam (eds.), Academic Press, New York, 1965, p. 229.

[15] A. Barone, G. Paterno, Physics and Applications of the Josephson effect, Wiley, New-York, 1982. 